\title{
MEDIDAS MORFOMÉTRICAS E SUAS CORRELAÇÕES EM BÚFALOS DA RAÇA MURRAH CRIADOS EM MOJUII DOS CAMPOS, PARÁ
}

\author{
Thaís Emanuely dos Santos Amaral'; Aline Pacheco²; Jéssica de Carvalho Pantoja ${ }^{3}$; Cristiane Rebouças \\ Barbosa'; Eliana Torres dos Nascimento ${ }^{5}$; Kedson Alessandri Lobo Neves ${ }^{6}$; Adcleia Pereira Pires ${ }^{7}$; Luan \\ Borges Souza ${ }^{8}$.

\footnotetext{
${ }^{1}$ Universidade Federal do Oeste do Pará, Santarém, Pará, Brasil, t.emanuely95@gmail.com 2Universidade Federal do Oeste do Pará, Santarém, Pará, Brasil, jessicka.carvalho17@gmail.com 3Universidade Federal do Oeste do Pará, Santarém, Pará, Brasil, cris_ag10@hotmail.com 4Universidade Federal do Oeste do Pará, Santarém, Pará, Brasil, elianaacademico@hotmail.com 5Universidade Federal do Oeste do Pará, Santarém, Pará, Brasil, piresadcleia@gmail.com. 6Universidade Federal do Oeste do Pará, Santarém, Pará, Brasil, alinepacheco@outlook.com 7 Universidade Federal do Oeste do Pará, Santarém, Pará, Brasil, kedson_neves@hotmail.com. 8Universidade da Amazônia, Santarém, Pará, Brasil, Brasil, luanmedvet90@gmail.com
}

RESUMO: Objetivou-se verificar o perfil morfométrico e suas correlações em búfalos (Bubalus bubalis) da raça Murrah no período de três meses no município de Mojuí dos Campos. Avaliaram-se 29 fêmeas com idade média de 36 meses e 7 machos inteiros com idade média de 18 meses, mensurando o perímetro torácico (PT), perímetro do barril (PB), comprimento do corpo (CC), altura de cernelha (AC), altura da garupa (AG), e peso corporal. Os dados foram tabulados de acordo com o sexo, sendo as médias comparadas pelo teste SNK, e as correlações entre as características através do procedimento PROC CORR. Todas as características analisadas apresentaram diferença significativa entre machos e fêmeas, sendo que as fêmeas apresentaram resultados superiores. Resultado este atribuído a diferença de idade, já que os machos eram animais jovens, com idade média de 18 meses, enquanto as fêmeas em estudo apresentaram média de idade de 36 meses. Machos e fêmeas da raça Murrah criados em Mojuí dos Campos apresentam medidas morfométricas diferentes. As correlações contatadas foram altas e favoráveis entre todas as variáveis analisadas. As características morfométricas que tiveram maior correlação com o peso foram o perímetro do barril $(r=0,95)$ e o perímetro torácico $(r=0,87)$, sendo estas as melhores medidas para predizer o peso vivo. A largura da garupa foi a medida com menor correlação $(r=0,49)$. Há poucos estudos de análise do padrão morfométrico em bubalinos na região, por isso torna-se necessário novas pesquisas para melhor conhecimento dos animais criados na região.

PALAVRAS-CHAVE: Biometria, Medidas corporais, Melhoramento genético. 


\title{
MORPHOMETRIC MEASUREMENTS AND THEIR CORRELATIONS IN THE MURRAH BUFFALOES RAISED IN MOJUÍ DOS CAMPOS, PARÁ
}

\begin{abstract}
The aim of this work was to analyze the morphometric aspects and their correlations in buffaloes (Bubalus bubalis) of the Murrah breed in the period of three months in the municipality of Mojuí dos Campos, state of Pará. Twenty-nine females with a mean age of 36 months and 7 males with a mean age of 18 months were evaluated, measuring body weight, chest circumference (PT), barrel perimeter (PB), body length (CC), height of withers (AC) and height of croup (AG). The statistic of the tabulated data was according to the analysis of variance and the means were compared by the SNK Proc. GLM. All the characteristics analyzed showed a significant difference between males and females, and females showed superior results. This result was attributed to the age difference, since the males were young animals, with an average age of 18 months, while the females under study had a mean age of 36 months. Positive correlations were observed in all analyzed variables. The morphometric characteristics that had the greatest correlation with the weight were the perimeter of the barrel $(r=0,95)$ and the thoracic perimeter $(r=0,87)$, being these the best measures to predict the live weight. The saddle width $(r=0,49)$ was the measure with the lowest correlation.
\end{abstract}

KEYWORDS: Biometry, Body measurements, Genetic improvement.

\section{MEDIDAS MORFO MÉTRICAS Y SUS CORRELACIONES EN BÚFALOS DE LA RAZA MURRAH CRIADOS EN MOJUÍ DOS CAMPOS, PARÁ}

RESUMEN: El objetivo de este trabajo fue verificar el perfil morfo métrico y sus correlaciones en búfalos (Bubalus bubalis) de raza Murrah, en el período de tres meses en el municipio de Mojuí dos Campos, Pará. Se evaluaron 29 hembras con edad media de 36 meses y 7 machos enteros con edad media de 18 meses, midiendo el perímetro torácico $(\mathrm{PT})$, perímetro de barril $(\mathrm{PB})$, largura del cuerpo $(\mathrm{LC})$, altura a la cruz $(A C)$, altura del anca (AA) y peso corporal. Los datos fueron tabulados de acuerdo con sexo, siendo las medias comparadas por el test SNK, y las correlaciones entre las características a través del procedimiento PROC CORR (SAS, 2006). Todas las características analizadas presentaron diferencia significativa entre machos y hembras, siendo que las hembras presentaron resultados superiores. Resultado este atribuido a la diferencia de edad, ya que los machos eran animales jóvenes, con una edad media de 18 meses, mientras que las hembras en estudio presentaron un promedio de edad de 36 meses. Correlaciones altas y favorables fueron constatadas entre todas las variables analizadas. Las características morfo métricas que tuvieron mayor correlación con el peso fueron el perímetro de barril $(r=0,95)$ y perímetro torácico 
$(r=0,87)$, siendo estas las mejores medidas para predecir el peso vivo. La altura del anca fue la medida con menor correlación $(r=0,49)$. Hay pocos estudios de análisis del patrón morfo métrico en bufalinos en la región, por eso se hacen necesarias nuevas investigaciones para mejor conocimiento de los animales criados en la región.

PALABRAS CLAVE: Biometría, Medidas corporales, Mejora genética.

\section{INTRODUÇÃO}

Os búfalos foram introduzidos no Brasil a partir do final do século XIX, usualmente em pequenos lotes originários da Ásia, Europa (Itália) e Caribe, motivados muito mais pelo seu exotismo que por suas qualidades zootécnicas (BERNARDES, 2007). Segundo dados do Ministério da Agricultura (2015) a população de bubalinos no Brasil é de 1.189.069 cabeças, tendo o estado do Pará como maior produtor do país com um rebanho de aproximadamente 400 mil cabeças, seguido do estado do Amapá com aproximadamente 270 mil animais.

Taveira et al. (2016) afirmaram que os bubalinos são considerados animais de triplo propósito por se adaptarem a produção de carne, leite e ao trabalho. Além da carne, o leite e o queijo de búfalo também são produtos que despertam atenção no mercado nacional. No entanto, a bubalinocultura ainda encontra-se em desenvolvimento no país como uma alternativa rentável e saudável. Isto ocorre devido aos atributos fisiológicos inerentes à espécie, os bubalinos apresentam uma marcante superioridade frente aos bovinos. Este fator prevalece, por ser a espécie provida de rusticidade, precocidade e fertilidade, além de se adaptar facilmente aos diferentes ambientes (SANTOS et al., 2007).

A raça Murrah é considerada a de maior predominância devido à sua maior habilidade para produção de leite e seus constituintes, quando comparada com as outras no Brasil. É necessário melhorar ainda a eficiência desses animais, para a consolidação 
dessa atividade no Brasil. Para isto é indispensável o conhecimento do potencial produtivo e reprodutivo, além dos fatores genéticos e ambientais que influenciam as características que o representam (JUNIOR et al., 2014).

Com isto, o interesse por estudos sobre a influência de aspectos biométricos nas características reprodutivas das búfalas impulsiona o desenvolvimento de técnicas a serem empregadas em busca de melhor eficiência reprodutiva, principalmente com a redução do intervalo de partos (FERRAZ, 2012). O autor afirma ainda, que em relação aos aspectos morfométricos, existem poucas informações disponíveis na literatura para a espécie bubalina. A literatura cita que existem inúmeros trabalhos de estudo de crescimento por meio de avaliação de pesos, mas em relação a trabalhos que correlacionam características de produção com medidas corporais são escassos.

Segundo Pimentel et al. (2011) a avaliação morfológica de animais, é baseada nas relações entre as diversas regiões do corpo e o conjunto formado por elas, sendo considerado bem proporcionado, se as partes do corpo, observadas em conjunto, forem adaptadas à função a que ele se destina. Ramalho et al., (2013) em seu trabalho relataram que algumas medidas lineares como comprimento corporal, altura da cernelha e garupa, associadas ao peso corporal e ao perímetro torácico podem definir animais quanto ao tamanho, às exigências nutricionais e à maturidade fisiológica.

A utilização de medidas biométricas fornece informações para se relacionar e comparar as diversas regiões do corpo, e isto constitui um recurso básico para avaliação do animal vivo e da carcaça. Portanto, diferentes medidas biométricas foram criadas com o objetivo de se predizer estas características produtivas no animal vivo (CLEMENTE, 2012).

Alguns estudos com medidas biométricas apresentaram correlações positivas (LUCENA, 2015; JUNIOR, 
2006; ALVES, 2010). Clemente (2012) afirma ainda que estas correlações podem ser utilizadas como parâmetros para gerar elementos úteis para programas de melhoramento genético, implementando programas de seleção que utilizem de tais medidas sem a necessidade de se abater os animais para efetuar avaliações. A utilização das medidas biométricas em animais vivos pode ser vista como ferramenta, que permite conhecer o desenvolvimento das diferentes partes que compõem o exterior dos animais.

Dessa forma, objetivou-se avaliar o desenvolvimento corporal de bubalinos da raça Murrah, através de análises de medidas morfométricas e suas correlações.

\section{MATERIAL E MÉTODOS}

O experimento foi realizado na Fazenda Santo Antônio, localizada no município de Mojuí dos Campos Pará, latitude 02010'17 S e longitude 56 44'42" W, limitados pelos municípios de Santarém, Prainha, Alenquer, Belterra e Uruará (BORGES,
2015). Apresentando clima predominantemente quente e úmido, característicos de regiões tropicais.

Foram utilizados 36 búfalos da raça Murrah, 29 fêmeas, com idade média de 36 meses e 7 machos inteiros com idade média de 18 meses. Os animais permaneciam em sistema de produção extensiva, com fornecimento de água e premix mineral à vontade. Era disponibilizado, uma vez ao dia, milho e sorgo, de acordo com a categoria e o estádio fisiológico dos animais.

O experimento foi realizado no período menos chuvoso, entre os meses de julho à setembro de 2017, sendo as mensurações realizadas a cada 30 dias. Nesse período há menor incidência de chuvas na região e as temperaturas do ar estão mais elevadas.

Os animais eram contidos e pesados em jejum, em balança mecânica do tipo tronco - balança. Após as pesagens, realizava-se, com auxílio de uma fita métrica graduada em centímetros, as seguintes medidas (PACHECO et al., 2008): Perímetro 
torácico (PT) - medida tomada pelo contorno do tórax passando pelo cilhadouro e voltando perpendicularmente à linha do dorso; Perímetro do barril (PB) - tomada ao redor da cavidade abdominal, na altura do rúmen; Comprimento do corpo (CC)- distância entre a extremidade anterior da espádua e a ponta ou extremidade posterior da nádega. E com auxílio de um hipômetro obtevese as medidas da Altura da garupa (AG) - vertical baixada do ângulo superior da garupa ao solo e da Altura da cernelha (AC) - tomada do ponto mais alto da cernelha ao solo e Largura da garupa (LG) - distância entre os ângulos externos das articulações coxofemorais (Figura 1).

Figura 1. Representação das medidas corporais. A) Altura e largura da garupa; B) Altura da cernelha.

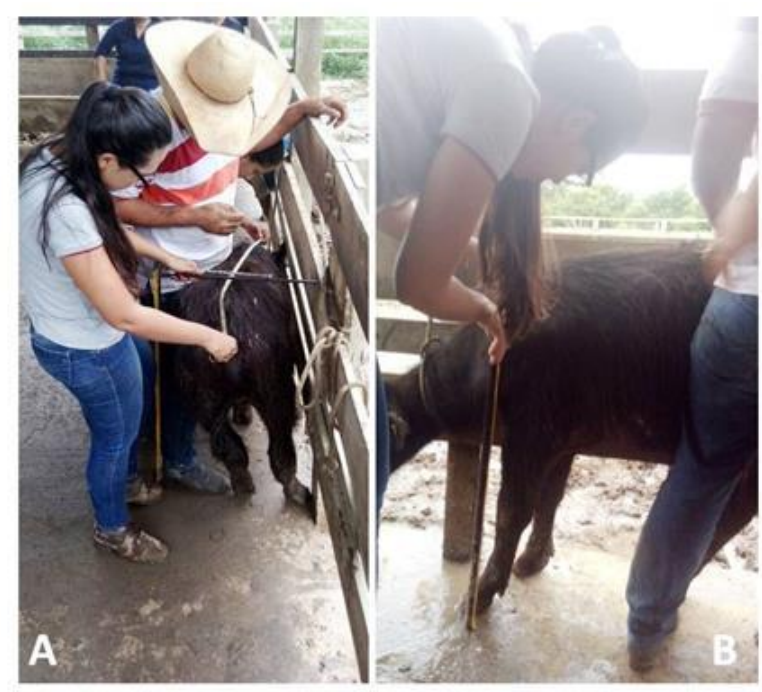

Para realizar as análises estatísticas dos dados tabulados utilizou-se $\mathrm{O}$ software SAS Learning Edition $4.1{ }^{\circledR}$ (SAS Institute, 2006).
A análise de variância para detectar os efeitos de sexo sobre as características morfométricas incluiu os fixos de fazenda e idade do animal. As 
médias foram comparadas pelo teste SNK, a 5\%, Proc. GLM e as correlações simples entre as características corporais pelo Proc. CORR (SAS, 2006).

\section{RESULTADOS E DISCUSSÃO}

Os valores médios e desvios padrão das medidas morfométricas da raça Murrah, fêmeas e machos, encontramse na Tabela 1. Observa-se que em todas as características analisadas houve diferença significativa $(P<0,05 \%)$ entre machos e fêmeas, sendo que as fêmeas apresentaram resultados superiores. Resultado este atribuído a diferença de idade, já que os machos eram animais jovens, com idade média de 18 meses, enquanto as fêmeas em estudo apresentaram média de idade de 36 meses.

Tabela 1. Médias e desvios padrão das características morfométricas de búfalos Murrah criados em Mojuí dos Campos/Pará.

\begin{tabular}{cccc}
\hline Características & Fêmeas $(\mathrm{n}=112)$ & Machos $(\mathrm{n}=23)$ & CV\% \\
\hline AC (cm) & $127 \pm 1,40^{\mathrm{a}}$ & $108 \pm 1,30^{\mathrm{b}}$ & 11,56 \\
AG (cm) & $131 \pm 1,30^{\mathrm{a}}$ & $114 \pm 4,0^{\mathrm{b}}$ & 10,16 \\
PT (cm) & $193 \pm 2,80^{\mathrm{a}}$ & $146 \pm 3,80^{\mathrm{b}}$ & 16,30 \\
PB (cm) & $218 \pm 2,90^{\mathrm{a}}$ & $160 \pm 3,90^{\mathrm{b}}$ & 15,03 \\
CC(cm) & $151 \pm 1,60^{\mathrm{a}}$ & $124 \pm 2,10^{\mathrm{b}}$ & 11,44 \\
LG (cm) & $62 \pm 1,80^{\mathrm{a}}$ & $43 \pm 1,00^{\mathrm{b}}$ & 29,75 \\
PESO (kg) & $436,00 \pm 120,50^{\mathrm{a}}$ & $247,64 \pm 109,13^{\mathrm{b}}$ & 28,83 \\
\hline
\end{tabular}

Letras diferentes na mesma linha diferem entre si pelo teste SNK a 5\% de probabilidade. Onde: AC Altura da Cernelha; AG - altura da Garupa; PT - perímetro torácico; PB - perímetro do barril; CC comprimento do corpo; LG - largura da Garupa; CV- coeficiente de variação.

Os valores médios de altura da castrados e inteiros da raça cernelha (AC) e perímetro torácico (PT) Mediterrâneo, criados na Baixada foram próximos ao encontrado por Litorânea do Rio de Janeiro, com Ramalho et al., (2013) em machos médias de 126,60 cm e 190,10 cm 
respectivamente. Enquanto que a altura da garupa (AG) encontrada pelo referido autor foi superior ao relatado neste trabalho, com valor de 125,80 cm. Os valores médios de perímetro torácico (PT) e altura da garupa foram menores que os relatados por Taveira et al., (2016) em fêmeas da raça Murrah $(219,87 \mathrm{~cm} \quad$ e $147,25 \mathrm{~cm})$ respectivamente. O resultado médio do peso dos animais encontrado neste trabalho foi menor que o encontrado por Oliveira et al., (2001) em búfalas mestiças $(509,7 \mathrm{~kg})$ e por Ramalho et al., (2013) em búfalos machos Mediterrâneo (452,10kg).

De acordo com Andrade e Garcia (2005) o peso médio padrão da raça Murrah é de 550 quilos (kg) para fêmeas e 750 kg nos machos. Segundo estes dados os valores médios de peso encontrados neste experimento para as fêmeas está abaixo do estimado para a raça, o valor médio de peso dos machos também se encontra abaixo do esperado, no entanto, deve-se levar em consideração que os búfalos machos utilizados neste trabalho, eram jovens com idade média de 18 meses, indicando que estes estariam ainda em processo de desenvolvimento corporal. O baixo peso das fêmeas e dos machos também pode ser resultado do manejo alimentar e da menos qualidade das forragens durante o período menos chuvoso da região.

Dentre as medidas biométricas a altura da cernelha, altura da garupa e comprimento do corpo foram as variáveis que apresentaram menor coeficiente de variação. Em relação a $A C$ e $A G$ foi menor provavelmente porque estas medidas foram obtidas por um hipômetro. Mesmo o CC sendo realizado por uma fita métrica também apresentou baixo coeficiente de variação, isso ocorreu devido à referência anatômica utilizada para obtenção desta medida morfométrica, a qual sofre pouquíssima variação do posicionamento do animal.

Correlações significativas foram encontradas entre todas as variáveis avaliadas de acordo com os dados da Tabela 2. Estas correlações podem ser utilizadas como parâmetros para gerar 
elementos úteis para programas de melhoramento genético, implementando projetos de seleção que utilizem de tais medidas sem a necessidade de se abater os animais para efetuar avaliações. A correlação alta e favorável entre perímetro torácico e o peso vivo, pode ser utilizada, por exemplo, para confecção de instrumentos métricos para aferição do peso vivo, facilitando o controle ponderal em inúmeras propriedades rurais que não dispõem de balança (CLEMENTE, 2012). Seixas (2006) afirmou que a seleção praticada atualmente enfatiza muito o tamanho corporal, dentre elas o comprimento corporal, a altura de garupa e altura de cernelha, pois estão diretamente relacionados com o peso do animal.

Tabela 2. Coeficiente de correlação de Pearson ( $r$ ) entre as medidas morfométricas e peso de búfalos Murrah criados em Mojuí dos Campos/Pará.

\begin{tabular}{lllllll}
\hline & AC & AG & PT & PB & CC & LG \\
\hline AC & - & & & & & \\
AG & 0,80 & - & & & & \\
PT & 0,73 & 0,76 & - & & & \\
PB & 0,79 & 0,82 & 0,91 & - & & \\
CC & 0,73 & 0,77 & 0,76 & 0,80 & - & - \\
LG & 0,54 & 0,58 & 0,54 & 0,60 & 0,55 & -49 \\
PESO & 0,78 & 0,78 & 0,87 & 0,95 & 0,77 & 0,49 \\
\hline
\end{tabular}

Pearson, * $P<0,01$. AC Altura da Cernelha; AG altura da Garupa; PT perímetro toráxico; PB perímetro do barril; CC comprimento do corpo; LG largura da Garupa

As correlações entre peso corporal e as medidas de comprimento do corpo e perímetro do barril nos búfalos apresentaram-se altas, positivas e significativas (entre $r=0,77$ e 0,95) $(P<0,01)$, enquanto que entre largura da garupa com as outras características os valores foram menores $(r=0,54$ e 0,60). o perímetro do barril e o perímetro torácico foram as medida com maior correlação com o peso corporal, indicando que estas podem ser as melhores características para estimar o peso vivo. Corroborando com estes resultados Alves (2010), utilizando as medidas entre idade, peso, perímetro 
torácico, comprimento corporal e altura da cernelha, em experimento com búfalos, demonstrou que todas as correlações avaliadas mostraram-se altamente significativas, o autor afirma ainda que a medida de perímetro torácico como a melhor característica para estimar o peso de búfalo, reafirmando 0 encontrado neste trabalho. Júnior et al., (2005) relataram que as variáveis fenotípicas comprimento corporal, perímetro torácico e largura de garupa são as melhores indicadoras de peso vivo, com destaque para o perímetro torácico.

A correlação entre peso corporal e altura da cernelha e altura da garupa foram iguais $(r=0,78)$, podendo juntamente com outras características serem indicadores do peso vivo, com exceção da característica largura da garupa que mostrou menor correlação $(r=0,49)$. Assim, búfalos mais altos no anterior e posterior, mais compridos, com maior perímetro torácico, garupa mais comprida e larga, tendem a ter maior peso. Com base nesse aspecto, podem ser criadas equações que permitam a utilização do perímetro torácico para predição de peso vivo de búfalos da raça Murrah, na inexistência de equipamentos de pesagem apropriados, nas propriedades rurais (JÚNIOR et al., 2005).

\section{CONCLUSÃO}

Machos e fêmeas da raça Murrah criados em Mojuí dos Campos, Pará apresentam medidas morfométricas diferentes. As correlações entre peso e medidas morfométricas foram altamente significativas, indicando que outras medidas além do peso podem ser utilizadas como característica auxiliar no processo de seleção de búfalas da raça Murrah. Devido a falta de informações sobre medidas morfométricas e correlaç̃̃es para a raça Murrah na literatura, torna-se necessário maiores estudos e novas pesquisas para melhor conhecimento dos animais criados na região.

\section{REFERÊNCIAS}

ALVES, T. C. Desenvolvimento ponderal, características da carcaça e eficiência da 
nutrição energética e proteica no metabolismo ruminal de búfalos e produção de gases in vitro, Pirassununga, 2010. Tese (Doutorado em Zootecnia)- Faculdade de Zootecnia e Engenharia de Alimentos da Universidade de São Paulo, 2010.

ANDRADE, V. J.; GARCIA, S. K. Padrões raciais e registros de bubalinos. Revista Brasileira de Reprodução Animal. v.29, n.1, p.39-45, 2005.

BERNARDES, O. Bubalinocultura no Brasil: situação e importância econômica. Revista Brasileira de Reprodução Animal. v.31, n.3, p.293298, 2007.

BORGES, T. C. S.; PEREIRA, I. C. N. Análise do uso múltiplo da água na bacia hidrográfica do rio Mojuí (PA): um esforço de sistematização. Revista GeoAmazônia, v. 3, n. 6, p. 42 - 53, 2015.

CLEMENTE, C. A. A. Avaliação do desenvolvimento biométrico e rendimento de cortes de cabritos das raças de cabritos das raças Saanen e Alpina, Belo Horizonte, 2012. Dissertação (Mestrado em Produção Animal)- Universidade Federal de Minas Gerais, Programa de Pós-Graduação em Zootecnia, Belo Horizonte, 2012.

FERRAZ, P. C. Biometria das características produtivas, reprodutivas e estrutural populacional de búfalos (Bulalus bubalis) explorados no Brasil, Itapetinga, 2012. Tese (Doutorado em Produção de Ruminantes)-
Universidade Estadual do Sudoeste da Bahia, Itapetinga, 2012.

JÚNIOR, G. S. C; CAMPELO, J. E. G; AZEVEDO, D. M. M. R; FILHO, R. M; CAVALCANTE， R. R; LOPES, J. B; OLIVEIRA, M. E. Caracterização morfométrica de ovinos da raça Santa Inês criados nas microrregiões de Teresina e Campo Maior, Piauí. Semina: Ciências Agrárias, Londrina, v. 36, n. 1, p. 353-366, 2015.

JUNIOR, J. B. L.; COSTA, N. A.; ARAUJO, C. V.; DUTRA, S.;GARCIA, A. R.; NAHUM, B. S.; MATOS, J. C. S.; BRANDAO, L. M. Sistema silvipastoril na produção sustentável de búfalos para carne na pequena propriedade da Amazônia oriental. Disponível em: http://www.bioclimatologia.ufc.br/loure nco.pdf. Acesso em: 22 out. 2017.

JUNIOR, J. S. B; FRAGA, A. B; COUTO, A. G; BARROS, C. C; SILVA, R. M. O. Produção de leite, duração da lactação e intervalo de partos em búfalas mestiças Murrah. Revista Caatinga, Mossoró, v. 27, n. 2, p. 184-191, 2014.

LUCENA, J. E. C; VIANNA, S. A. B; ROMERO, F. B. N; FILHO, L. M. S; DINIZ, W. J. S. Estudo comparativo das proporções morfométricas entre garanhões e castrados da raça Campolina. Revista Brasileira de Zootecia. v. 35, n. 6, p. 2260-2267, 2006.

MINISTÉRIO DA AGRICULTURA, PECUÁRIA E ABASTECIMENTO. MAPA. Dados de Rebanho bovino e bubalino do Brasil, 2015. Disponível em: http://www.agricultura.gov.br/assuntos/ 
sanidade-animal-e-vegetal/saude-

animal/programas-de-saude-

animal/copy

of

DadosdeRebanhobovinoebubalinodoB rasil 2015.pdf. Acesso em: 21 out. 2017.

MENEZES. L. F. G. et al. Medidas corporais de novilhos das gerações avançadas do cruzamento rotativo entre as raças Charolês e Nelore, terminados em confinamento. Ciência Rural, v.38, n.3, p.771-777, 2008

OLIVEIRA, C. A.; BOMBONATO, P. P.; BARUSELLI, P. S.; OLIVEIRA, J. F. S.; SOUZA, A. O. Pelvimetria e pelvilogia em búfalas mestiças (Bubalus bubalis).

Brazilian Journal of veterinary Research animal Science. v. 38, n. 3, p. 114-121, 2001.

PACHECO, A.; QUIRINO, C. R.; PINHEIRO, O. L. V. M.; ALMEIDA, J. V. C. Medidas morfométricas de touros jovens e adultos da raça Guzerá. Revista Brasileira de Saúde e Produção Animal. v. 9, n. 3, p. 426-435, 2008.

PIMENTEL, M. M. L; CÂMARAL, F. V; DANTAS, R. A; FREITAS, Y. B. N; DIAS, R. V. C; SOUZA, M. V. Biometria de equinos de vaquejada no Rio Grande do Norte, Brasil. Acta Veterinaria Brasilica, v. 5, n. 4, p. 376-379, 2011.

RAMALHO, R. O. S; RODRIGUES, V. C; COUTO, D. M; PITOMBO, R. S; SOUZA,
D. D. N; ARAujo, A. H. B. Medidas corporais e características de carcaça de bubalinos mediterrâneos castrados e inteiros. Boletim Indústria animal. v. 70, n. 1, p. 20-27, 2013.

SANTOS, D. A. S.; RICCl, R. E. G.; PRADO, I. M. M. I; CARVALHO, A. F.; BOMBONATO, P. P.; AMBROSIO, C. E.; TEXEIRA, D. G.; SANTOS, T. C.; BRAZQUEZ, F. J. H.; MARTINS, D. S.; MORINI, A. C.; JUNIOR, J. R. K.; MIGLINO, M. A. Morfologia e morfometria das papilas mamárias de búfalas. Pesquisa Veterinária Brasileira. v.27, n., 3, 2007.

SAS Institute. Statistical Analysis System. SAS Learning Edition 4.1®, SAS Institute Inc, 2006.

SEIXAS, V. N. C. Determinação de parâmetros para a avaliação de carcaça em bubalinos machos no estado do Pará, Belém, 2006. Dissertação (Mestrado em Ciência Animal) Universidade Federal do Pará, Centro de Ciências Agrárias, Núcleo de Estudos em Ciência Animal, 2006.

TAVEIRA, R. Z; AMARAL, A. G; CARVALHO, F. E; MARTINS, T. R; NETO, O. J. S; CAMPOS, J. C. D. Avaliaçãao de característica de tipo e produção de leite em rebanho bubalino. Revista Espacios, v. 38, n. 13, p. 22-26, 2016. 